\title{
L2 Gain Stability Analysis of Event-triggered Agreement Protocols
}

\author{
Dimos V. Dimarogonas
}

\begin{abstract}
We extend our previous results on event-triggered agreement by proposing a triggering mechanism that is finite $\mathcal{L}_{2}$ gain stable with respect to additive disturbances in the open loop dynamics of the agents. Moreover, the control design is both distributed and provides strictly positive inter-execution times. Simulation examples support the derived theoretical results.
\end{abstract}

\section{INTRODUCTION}

The control of distributed multi-agent systems is now facilitated by the current increase in computing and communication resources. Several results concerning multi-agent cooperative control have appeared in recent literature involving consensus algorithms [13],[14],[10] formation control [4],[3],[2] and distributed estimation [16].

As the number of agents increases, there is a need for an optimized allocation of the available resources. This paper provides another potential contribution towards this direction. In particular, this work is concerned with the adaptation of near-optimal sampling strategies for the actuators in a distributed multi-agent system that aims at achieving agreement. The scheduling of the actuation updates can be done in a time-driven or an event-driven fashion. It is possible that an intelligent strategy for sampling will provide a better allocation of available resources. Motivated by this assertion, in recent work [5],[6],[15] we applied the framework of event-based control [17],[20],[19],[9],[1],[12] to cooperative control of multi-agent systems, and more specifically, to the well-studied case of agreement or consensus distributed control design.

This work builds upon the results of [5],[15] in a twofold manner. While [5] provided a purely distributed event-based strategy, there were no guarantees that all the agents had a strictly positive inter-execution time. This is avoided here by redefining the non-cooperative event-triggering rules of [15] in a cooperative manner. Thus the resulting event-based controllers can be seen as a combination of the frameworks presented in our previous work in [5],[15]. The first result of the paper establishes that the distributed event-based control strategy guarantees a strict lower bound on the interexecution times. We then proceed to the main result of the paper that proves finite $\mathcal{L}_{2}$ gain stability for the case of additive noise in each of the agents' dynamics in the open loop system. Stability to an arbitrarily small set around the

Dimos Dimarogonas is with the KTH ACCESS Linnaeus Center, School of Electrical Engineering, Royal Institute of Technology (KTH), Stockholm, Sweden $\{$ dimos@ee.kth.se . He is also affiliated with the KTH Center of Autonomous Systems (CAS) and is supported by the Swedish Research Council (VR) through contract 2009-3948. This work was also supported by the Swedish Research Council, the EU HYCON2 NoE, and the EU FeedNetBack STREP FP7 project. agreement point is guaranteed in both cases. The results utilize the framework of [18], where finite $\mathcal{L}_{2}$ gain stability was tackled for interconnected linear systems with noncooperative equilibria. In contrast, the results of the current paper involve cooperative equilibria, i.e., convergence to the agreement manifold.

The rest of the paper is organized as follows: Section II presents some necessary background and discusses the problem treated in the paper. The new event-triggered ruling is discussed in Section III while Section IV presents the analysis of the finite $\mathcal{L}_{2}$ gain stability for the perturbed case. Some examples are given in Section V while Section VI includes a summary of the results of this paper.

\section{BACKground And Problem Statement}

In this section we first review some related results on algebraic graph theory [8] that are used in the paper and proceed to describe the problem in hand.

\section{A. Algebraic Graph Theory}

For an undirected graph $G$ with $N$ vertices the adjacency matrix $A=A(G)=\left(a_{i j}\right)$ is the $N \times N$ matrix given by $a_{i j}=1$, if $(i, j) \in E$, where $E$ is the set of edges, and $a_{i j}=0$, otherwise. If there is an edge $(i, j) \in E$, then $i, j$ are called adjacent. A path of length $r$ from a vertex $i$ to a vertex $j$ is a sequence of $r+1$ distinct vertices starting with $i$ and ending with $j$ such that consecutive vertices are adjacent. For $i=j$, this path is called a cycle. If there is a path between any two vertices of the graph $G$, then $G$ is called connected. A connected graph is called a tree if it contains no cycles. The degree $d_{i}$ of vertex $i$ is defined as the number of its neighboring vertices, i.e. $d_{i}=\{\# j:(i, j) \in E\}$. Let $\Delta$ be the $n \times n$ diagonal matrix of $d_{i}$ 's. Then $\Delta$ is called the degree matrix of $G$. The (combinatorial) Laplacian of $G$ is the symmetric positive semidefinite matrix $L=\Delta-A$. For a connected graph, the Laplacian has a single zero eigenvalue and the corresponding eigenvector is the vector of ones, 1 . We denote by $0=\lambda_{1}(G) \leq \lambda_{2}(G) \leq \ldots \leq \lambda_{N}(G)$ the eigenvalues of $L$. If $G$ is connected, then $\lambda_{2}(G)>0$. An orientation on $G$ is the assignment of a direction to each edge. An oriented graph has the the incidence matrix $B=$ $B(G)=\left(b_{i j}\right)$, which is the $\{0, \pm 1\}$ matrix with rows and columns indexed by the vertices and edges of $G$, respectively, such that $b_{i j}=1$ if the vertex $i$ is the head of the edge $j$, and $b_{i j}=-1$ if vertex $i$ is the tail of the edge $j$, and $b_{i j}=$ 0 otherwise. Obviously, the matrix $B$ varies with different assignment of the edges' orientation. The Laplacian matrix is also given by $L=B B^{T}$. 


\section{B. System Model}

The system considered consists of $N$ agents, with $x_{i} \in \mathbb{R}$ denoting the state of agent $i$. Note that the results of the paper are extendable to arbitrary dimensions. We assume that agents' motion obeys a single integrator model:

$$
\dot{x}_{i}=u_{i}, i \in \mathcal{N}=\{1, \ldots, N\}
$$

where $u_{i}$ denotes the control input for each agent.

Each agent is assigned a subset $N_{i} \subset\{1, \ldots, N\}$ of the rest of the team, called agent $i$ 's communication set, that includes the agents with which it can communicate. The undirected communication graph $G=\{V, E\}$ of the multiagent team consists of a set of vertices $V=\{1, \ldots, N\}$ indexed by the team members, and a set of edges, $E=$ $\left\{(i, j) \in V \times V \mid i \in N_{j}\right\}$ containing pairs of vertices that correspond to communicating agents. Moreover, we denote by $\bar{x}$ the $m$-dimensional stack vector of relative differences of pairs of agents that form an edge in $G$, where $m$ is the number of edges. The following relations are easily verified: $L x=B \bar{x}, \bar{x}=B^{T} x$. Since $\bar{x}=0 \Rightarrow B \bar{x}=0 \Rightarrow L x=0$, then if $G$ is connected, the requirement $L x=0$ guarantees that $x$ has all its elements equal.

\section{Problem Statement}

The agreement control laws in [7],[13] were given by

$$
u_{i}=-\sum_{j \in N_{i}}\left(x_{i}-x_{j}\right)
$$

and the closed-loop equations of the nominal system were $\dot{x}_{i}=-\sum_{j \in N_{i}}\left(x_{i}-x_{j},\right), i \in\{1, \ldots, N\}$, so that $\dot{x}=-L x$, where $x=\left[x_{1}, \ldots, x_{N}\right]^{T}$ and $L$ is the graph Laplacian. For a connected graph, all agents' states converge to a common point, called the "agreement point", which coincides with the average $\frac{1}{N} \sum_{i} x_{i}(0)$ of the initial states.

In [6],[5] we redefined the above control formulation to take into account distributed event-triggered strategies. In particular, for each $i \in \mathcal{N}$, and $t \geq 0$, introduce a (state) measurement error $e_{i}(t)$. Denote the stack vector $e(t)=\left[e_{1}(t), \ldots, e_{N}(t)\right]^{T}$. A sequence of events $t_{0}^{k}, t_{1}^{k}, \ldots$ is defined for each agent $k$ according to $f_{k}\left(e_{k}\left(t_{i}^{k}\right), \sum_{j \in N_{k}}\left(x_{i}\left(t_{i}^{k}\right)-x_{j}\left(t_{i}^{k}\right)\right)\right)=0$, for $k \in \mathcal{N}$ and $i=0,1, \ldots$. Hence a condition encoded by the function $f_{k}\left(e_{k}\left(t_{i}^{k}\right), \sum_{j \in N_{k}}\left(x_{i}\left(t_{i}^{k}\right)-x_{j}\left(t_{i}^{k}\right)\right)\right)$ triggers the events for agent $k \in \mathcal{N}$. The decentralized control law for $k$ is updated both at its own event times $t_{0}^{k}, t_{1}^{k}, \ldots$, as well as at the last event times of its neighbors $t_{0}^{j}, t_{1}^{j}, \ldots, j \in N_{k}$. Thus it is of the form

$$
u_{k}(t)=u_{k}\left(t_{i}^{k}, \bigcup_{j \in N_{k}} t_{i^{\prime}(t)}^{j}\right),
$$

where $i^{\prime}(t) \triangleq \arg \min _{l \in \mathbb{N}: t \geq t_{l}^{j}}\left\{t-t_{l}^{j}\right\}$. We aim at deriving control laws of the form (3), and event times $t_{0}^{k}, t_{1}^{k}, \ldots$, for each agent $k \in \mathcal{N}$ that drive (1) to an agreement point.

\section{NeW EVENT-TRIGGERED RULES FOR THE DECENTRALIZED APPROACH}

In this section we redefine the triggering rules of [5] in the decentralized case to guarantee that there is a strict minimum inter-execution time for all agents. In the decentralized eventtriggered cooperative control formulation, each agent updates its own control input at event times it decides based on information from its neighboring agents. The event times for each agent $i \in \mathcal{N}$ are denoted by $t_{0}^{i}, t_{1}^{i}, \ldots$

The measurement error for agent $i$ is defined as

$$
e_{i}(t)=x_{i}\left(t_{k}^{i}\right)-x_{i}(t), t \in\left[t_{k}^{i}, t_{k+1}^{i}\right)
$$

The decentralized control strategy for agent $i$ is:

$$
u_{i}(t)=-\sum_{j \in N_{i}}\left(x_{i}\left(t_{k}^{i}\right)-x_{j}\left(t_{k^{\prime}(t)}^{j}\right)\right)
$$

where

$$
k^{\prime}(t) \triangleq \arg \min _{l \in \mathbb{N}: t \geq t_{l}^{j}}\left\{t-t_{l}^{j}\right\}
$$

Note that we have $x_{j}\left(t_{k^{\prime}(t)}^{j}\right)=x_{j}(t)+e_{j}(t)$, so that $\dot{x}_{i}(t)=-\sum_{j \in N_{i}}\left(x_{i}\left(t_{k}^{i}\right)-x_{j}\left(t_{k^{\prime}(t)}^{j}\right)\right)==$ $-\sum_{j \in N_{i}}\left(x_{i}(t)-x_{j}(t)\right)-\sum_{j \in N_{i}}^{j \in N_{i}}\left(e_{i}(t)-e_{j}(t)\right)$. Similarly to [13], the state vector $x$ can be decomposed as $x(t)=$ $\bar{x}(t) \mathbf{1}+\delta(t)$, where $\bar{x}(t)=\frac{1}{N} \sum_{i} x_{i}(t)$ denotes the average of the agents' states and $\delta$ is called the disagreement vector in [13] and $\mathbf{1}$ is the vector of ones. It can easily be shown that $\dot{\bar{x}}=0$ for the agents' initial average. Let us denote $\bar{x}(t)=\bar{x}(0)=\bar{x}$ for all $t \geq 0$. Note that the above control law can be written in stack vector form as $\dot{x}=-L(x+e)$. We have $\dot{x}=\dot{\delta}=-L(x+e)=-L(\bar{x} 1+\delta+e)$, so that

$$
\dot{\delta}=-L(\delta+e)
$$

For an undirected graph, an important property of $\delta$ proven in [13] is $\delta^{T} L \delta \geq \lambda_{2}(G)\|\delta\|^{2}$ for all $\delta$ satisfying $x=\bar{x} \mathbf{1}+\delta$.

Denote now $L x \triangleq z=\left[z_{1}, \ldots, z_{N}\right]^{T}$ and consider the Lyapunov function candidate

$$
V=\frac{1}{2} x^{T} L x
$$

It is shown in [5] that

$$
\dot{V} \leq-\sum_{i}\left(1-a\left|N_{i}\right|\right) z_{i}^{2}+\sum_{i} \frac{1}{a}\left|N_{i}\right| e_{i}^{2}
$$

where $a>0$. We will now redefine the triggering rule of [5] in order to achieve a strictly positive inter-execution time for all agents, and not only one agent at a time, as in [5]. The resulting event-triggering strategy can be considered as a combination of the triggering rules derived in our earlier papers [5] and [15].

Assume that $a$ satisfies

$$
0<a<\frac{1}{\left|N_{i}\right|}
$$


for all $i \in \mathcal{N}$. Then, enforcing the condition

$$
e_{i}^{2} \leq \frac{\sigma_{i} a\left(1-a\left|N_{i}\right|\right)}{\left|N_{i}\right|} z_{i}^{2}+\varepsilon_{i}
$$

where $\varepsilon_{i}>0$ is a scalar constant for all $i \in \mathcal{N}$, we get

$$
\dot{V} \leq \sum_{i}\left(\sigma_{i}-1\right)\left(1-a\left|N_{i}\right|\right) z_{i}^{2}+\sum_{i} \frac{1}{a}\left|N_{i}\right| \varepsilon_{i}
$$

The latter implies

$$
\dot{V} \leq-\min _{i}\left(1-\sigma_{i}\right)\left(1-a\left|N_{i}\right|\right)|| z \|^{2}+\sum_{i} \frac{1}{a}\left|N_{i}\right| \varepsilon_{i}
$$

which is negative for $0<\sigma_{i}<1$ and

$$
\|z\|^{2}>\frac{\sum_{i}\left|N_{i}\right| \varepsilon_{i}}{a \min _{i}\left(1-\sigma_{i}\right)\left(1-a\left|N_{i}\right|\right)}
$$

The additional term $\varepsilon_{i}>0$ in the event triggering rule (8) with respect to the one in [5] allows for guaranteeing a lower bound in the inter-execution times.

We can see now that for each $i \in \mathcal{N}$, an event is triggered when

$$
f_{i}\left(e_{i}, \sum_{j \in N_{i}}\left(x_{i}-x_{j}\right)\right) \triangleq e_{i}^{2}-\beta_{i} z_{i}^{2}-\varepsilon_{i}=0
$$

where $z_{i}=\sum_{j \in N_{i}}\left(x_{i}-x_{j}\right)$ and we also use the notation $\beta_{i}=\frac{\sigma_{i} a\left(1-a\left|N_{i}\right|\right)}{\left|N_{i}\right|}$. The update rule (9) holds at the event times $t_{k}^{i}$ corresponding to agent $i$ :

$$
f_{i}\left(e_{i}\left(t_{k}^{i}\right), \sum_{j \in N_{i}}\left(x_{i}\left(t_{k}^{i}\right)-x_{j}\left(t_{k}^{i}\right)\right)\right)=0
$$

with $k=0,1, \ldots$ and $i \in \mathcal{N}$. At an event time $t_{k}^{i}$, we have

$$
e_{i}\left(t_{k}^{i}\right)=x_{i}\left(t_{k}^{i}\right)-x_{i}\left(t_{k}^{i}\right)=0
$$

and thus, condition (8) is enforced.

It should be emphasized that the condition (9) is verified by agent $i$ only based on information of each own and neighboring agents' information.

The following theorem regarding the inter-event times holds:

Theorem 1: Consider system $\dot{x}_{i}=u_{i}, i \in \mathcal{N}=$ $\{1, \ldots, N\}$ with the control law (5) and update ruling (9), and assume that $G$ is connected. Suppose that $0<a<\frac{1}{\left|N_{i}\right|}$ and $0<\sigma_{i}<1$ for all $i \in \mathcal{N}$. Then for any initial condition in $\mathbb{R}^{N}$, and any time $t \geq 0$, as long as

$$
\|z\|^{2}>\frac{\sum_{i}\left|N_{i}\right| \varepsilon_{i}}{a \min _{i}\left(1-\sigma_{i}\right)\left(1-a\left|N_{i}\right|\right)},
$$

the inter-execution times of all agents are lower bounded by a strictly positive lower bound.

Proof: As long as

$$
\|z\|^{2}>\frac{\sum_{i}\left|N_{i}\right| \varepsilon_{i}}{a \min _{i}\left(1-\sigma_{i}\right)\left(1-a\left|N_{i}\right|\right)}
$$

we know that $\dot{V}<0$. Thus $V(t)<V(0)$ for all $t>0$ and this implies

$$
x^{T} L x=x^{T} B B^{T} x=\left\|B^{T} x\right\|^{2}<V(0)
$$

Note now that $L x=B B^{T} x$ so that $\|L x\| \leq\|B\|\left\|B^{T} x\right\|$, which implies $\|L x\| \leq\|B\| \sqrt{V(0)}$, i.e., $\|L x\|$ remains bounded.

Assume now that agent $i$ updates its control law at time $t^{*}$. Then $e_{i}\left(t^{*}\right)=0$ and $f_{i}\left(t^{*}\right) \leq-\varepsilon_{i}<0$, so that $i$ cannot trigger again instantaneously, i.e., Zeno behavior is excluded. We can now compute lower bounds on the interexecution times. In particular, since in between $t^{*}$ and the next update time of $i$ we have that $\dot{e}_{i}=-\dot{x}_{i}=-u_{i}$, we have $\left|e_{i}(t)\right| \leq$ $\int_{t^{*}}^{t}\left|u_{i}(s)\right| d s$ for all $t>t^{*}$ until the next update time of $i$.

From (8) we have that $\|e\|^{2}=\sum_{i}\left|e_{i}\right|^{2} \leq \sum_{i} \beta_{i} z_{i}^{2}+$ $\sum_{i} \varepsilon_{i}$. Using the notation $\bar{\beta}=\max _{i} \beta_{i}$ and $\bar{\varepsilon}=\sum_{i} \varepsilon_{i}$ the last equation yields

$$
\|e\|^{2} \leq \bar{\beta}\|L x\|^{2}+\bar{\varepsilon}
$$

We can then derive $\left|u_{i}\right| \leq\|u\|=\|L(x+e)\| \leq\|L x\|+$ $\|L\|(\sqrt{\bar{\beta}}|| L x \|+\sqrt{\bar{\varepsilon}})$, and, using $\|L x\| \leq\|B\| \sqrt{V(0)}$, we finally have

$$
\left.\left|u_{i}(t)\right| \leq\|B\| \sqrt{V(0)}(1+\|L\| \sqrt{\bar{\beta}})+\|L\| \sqrt{\bar{\varepsilon}}\right) \triangleq \bar{u}
$$

Since $\left|e_{i}(t)\right| \leq \int_{t^{*}}^{t}\left|u_{i}(s)\right| d s$ for all $t>t^{*}$, we have $\left|e_{i}(t)\right| \leq$ $\bar{u}\left(t-t^{*}\right)$, for all $t>t^{*}$ before the next event for agent $i$ is triggered. The next event is not triggered before (8) is violated, and this cannot happen before $\left|e_{i}(t)\right|=\sqrt{\varepsilon_{i}}$. A lower bound for the next event time is thus given by $t-t^{*}=$ $\frac{\sqrt{\varepsilon_{i}}}{\bar{u}}$. Therefore the minimum lower bound for the next event time for all agents $i$ and all times $t$ is given by $t-t^{*}=$ $\frac{\min _{i} \sqrt{\varepsilon_{i}}}{\bar{u}}$, or using the standard notation for the inter-event times, we have

$$
t_{k+1}^{i}-t_{k}^{i}=\frac{\min _{i} \sqrt{\varepsilon_{i}}}{\bar{u}}
$$

for all $i \in \mathcal{N}, k=0,1, \ldots \diamond$

We now check the convergence properties of the closed loop system (1),(5), (9). Following the derivations of [15], it can be shown that the disagreement vector $\delta(t)$ in (6) satisfies

$$
\|\delta(t)\| \leq e^{-\lambda_{2} t}|| \delta(0)\left\|+\int_{0}^{t} e^{-\lambda_{2}(t-s)}\right\| L e(s) \| d s
$$

where $\lambda_{2}$ is the second smallest eigenvalue of the Laplacian matrix of $G$ which satisfies $\lambda_{2}>0$ for a connected $G$. Remember that

$$
\|e\|^{2} \leq \bar{\beta}\|L x\|^{2}+\bar{\varepsilon}
$$

and assume that

$$
\|L x\|^{2}=\|z\|^{2} \leq \frac{\sum_{i}\left|N_{i}\right| \varepsilon_{i}}{a \min _{i}\left(1-\sigma_{i}\right)\left(1-a\left|N_{i}\right|\right)} \triangleq M_{1}
$$


Then $\delta(t)$ is bounded by

$$
\begin{aligned}
\|\delta(t)\| \leq e^{-\lambda_{2} t}(\|\delta(0)\| & \left.-\frac{\|L\|^{2} \sqrt{\bar{\beta} M_{1}+\bar{\varepsilon}}}{\lambda_{2}}\right)+ \\
& +\frac{\|L\|^{2} \sqrt{\bar{\beta} M_{1}+\bar{\varepsilon}}}{\lambda_{2}} \triangleq M_{2}(t)
\end{aligned}
$$

Note that $\dot{V}<0$ as long as $\|z\|^{2}>M_{1}$. Note also that $M_{1}$ is a linear function of $\varepsilon_{i}$ and that $M_{2}(t)$ contains the exponentially decaying term in $e^{-\lambda_{2} t}$. From (11) and $\delta=$ $x-\bar{x} \mathbf{1}$, we have that $\|z\|^{2} \leq M_{1}$ implies $V=x^{T} L x \leq$ $M_{1}\left(M_{2}(t)+\||\bar{x}|\right)$. We can now state the following result:

Corollary 2: Consider system $\dot{x}=u$ with the control law (5),(9) and assume that the communication graph $G$ is connected. Then the system reaches the time varying set $V=x^{T} L x \leq M_{1}\left(M_{2}(t)+\|\bar{x}\|\right)$ in finite time and remains within this set. As $t \rightarrow \infty$, the following bound holds:

$$
\|L x\|^{2} \leq\|B\|^{2} M_{1}\left(\frac{\|L\|^{2} \sqrt{\bar{\beta} M_{1}+\bar{\varepsilon}}}{\lambda_{2}}+\|\bar{x}\|\right)
$$

Proof: The first part is straightforward from the above analysis. For the second part, note that for $t \rightarrow \infty$, we have that $V(t) \leq M_{1}\left(\frac{\|L\|^{2} \sqrt{\bar{\beta} M_{1}+\bar{\varepsilon}}}{\lambda_{2}}+\|\mid \bar{x}\|\right)$. The result is now derived from the use of the relations $x^{T} L x=\left\|B^{T} x\right\|^{2}$ and $\|L x\| \leq\|B \mid\| B^{T} x \| \cdot \diamond$

\section{L2 GAIN ANALYSIS}

In this section we will examine the robustness of the proposed approach with respect to additive disturbances in the model. In particular, we assume that each agent's dynamics are perturbed by an additive noise of the form

$$
\dot{x}_{i}=u_{i}+w_{i}, i \in \mathcal{N}=\{1, \ldots, N\}
$$

We assume that each $w_{i}$ is a one-dimensional $\mathcal{L}_{2}$ function. The closed-loop dynamics for each agent $i$ are thus now given by

$$
\begin{aligned}
\dot{x}_{i}(t) & =-\sum_{j \in N_{i}}\left(x_{i}\left(t_{k}^{i}\right)-x_{j}\left(t_{k^{\prime}(t)}^{j}\right)\right)+w_{i}(t)= \\
& =-\sum_{j \in N_{i}}\left(x_{i}(t)-x_{j}(t)\right)-\sum_{j \in N_{i}}\left(e_{i}(t)-e_{j}(t)\right)+w_{i}(t)
\end{aligned}
$$

Note that the above control law can be written in stack vector form as

$$
\dot{x}=-L(x+e)+w
$$

where $w=\left[w_{1}, \ldots, w_{N}\right]^{T}$ is the stack vector of all disturbances.

We will examine the finite $\mathcal{L}_{2}$-gain stability [11] from the vector $L x$ that represents the agreement objective to the noise terms $w$ for the system (13).

Consider again the Lyapunov function candidate

$$
V=\frac{1}{2} x^{T} L x
$$

Then

$\dot{V}=x^{T} L \dot{x}=-x^{T} L(L x+L e-w)=-z^{T} z-z^{T} L e+z^{T} w$
From the definition of the Laplacian matrix we get

$$
\begin{aligned}
\dot{V} & =-\sum_{i} z_{i}^{2}-\sum_{i} \sum_{j \in N_{i}} z_{i}\left(e_{i}-e_{j}\right)+\sum_{i} z_{i} w_{i} \\
& =-\sum_{i} z_{i}^{2}-\sum_{i}\left|N_{i}\right| z_{i} e_{i}+\sum_{i} \sum_{j \in N_{i}} z_{i} e_{j}+\sum_{i} z_{i} w_{i}
\end{aligned}
$$

Using similar techniques as in the previous section, we can bound $\dot{V}$ as

$$
\begin{aligned}
\dot{V} \leq & -\sum_{i}\left(1-a\left|N_{i}\right|\right) z_{i}^{2}+\sum_{i} \frac{1}{a}\left|N_{i}\right| e_{i}^{2} \\
& +\sum_{i} \frac{\zeta}{2} z_{i}^{2}+\sum_{i} \frac{1}{2 \zeta} w_{i}^{2}
\end{aligned}
$$

where $a, \zeta>0$. Assume that the triggering condition is now given by

$$
e_{i}^{2} \leq \frac{\sigma_{i} a\left(1-a\left|N_{i}\right|-\zeta / 2\right)}{\left|N_{i}\right|} z_{i}^{2}+\varepsilon_{i}
$$

and denote by $\theta_{i} \triangleq \frac{\sigma_{i} a\left(1-a\left|N_{i}\right|-\zeta / 2\right)}{\left|N_{i}\right|}$. We can see now that for each $i \in \mathcal{N}$, an event is triggered when

$$
f_{i}\left(e_{i}, \sum_{j \in N_{i}}\left(x_{i}-x_{j}\right)\right) \triangleq e_{i}^{2}-\theta_{i} z_{i}^{2}-\varepsilon_{i}=0
$$

Then we have

$$
\begin{aligned}
\dot{V} \leq & -\sum_{i}\left(1-\sigma_{i}\right)\left(1-a\left|N_{i}\right|-\zeta / 2\right) z_{i}^{2} \\
& +\sum_{i} \frac{\left|N_{i}\right|}{a} \varepsilon_{i}+\sum_{i} \frac{1}{2 \zeta} w_{i}^{2}
\end{aligned}
$$

Assume that the controller parameters are chosen so that $0<\sigma_{i}<1$ and $0<a\left|N_{i}\right|+\zeta / 2<1$. Then the last inequality implies

$$
\begin{aligned}
\dot{V} \leq & -\min _{i}\left\{\left(1-\sigma_{i}\right)\left(1-a\left|N_{i}\right|-\zeta / 2\right)\right\}\|z\|^{2} \\
& +\sum_{i} \frac{\left|N_{i}\right|}{a} \varepsilon_{i}+\frac{1}{2 \zeta}\|w\|^{2}
\end{aligned}
$$

which shows that the closed loop system is finite $\mathcal{L}_{2^{-}}$ gain stable with an induced gain which is less than $\frac{1}{\sqrt{2 \zeta \min _{i}\left\{\left(1-\sigma_{i}\right)\left(1-a\left|N_{i}\right|-\zeta / 2\right)\right\}}}$. The preceding analysis is summarized in the following theorem:

Theorem 3: Consider system (13) with the triggering rule (14) and assume that the communication graph $G$ is connected. Assume that the controller parameters are chosen so that $0<\sigma_{i}<1$ and $0<a\left|N_{i}\right|+\zeta / 2<1$ for all $i \in \mathcal{N}$. Then the closed-loop system is finite $\mathcal{L}_{2}$-gain stable with an induced gain which is less than $\frac{1}{\sqrt{2 \zeta \min _{i}\left\{\left(1-\sigma_{i}\right)\left(1-a\left|N_{i}\right|-\zeta / 2\right)\right\}}}$.

Having established finite $\mathcal{L}_{2}$-gain stability, we will now derive similar lower bounds on the inter-execution times for each agent. Towards this goal, assume that there is a uniform upper bound in the noise terms in (12) of the form $w_{i}(t) \leq \bar{w}$ for all $i \in \mathcal{N}$ and all $t \geq 0$. 
The following result then holds:

Theorem 4: Consider the system (12) with the control law (5) and update ruling (14) and assume that the communication graph $G$ is connected. Suppose that $0<\sigma_{i}<1$ and $0<a\left|N_{i}\right|+\zeta / 2<1$ for all $i \in \mathcal{N}$ and $w_{i}(t) \leq \bar{w}$ for all $i \in \mathcal{N}$ and all $t \geq 0$. Then for any initial condition in $\mathbb{R}^{N}$, and any time $t \geq 0$, as long as

$$
z_{i}^{2}>\frac{\frac{\varepsilon_{i}\left|N_{i}\right|}{a}+\frac{\bar{w}}{2 \zeta}}{\left(1-\sigma_{i}\right)\left(1-a\left|N_{i}\right|-\zeta / 2\right)}
$$

for all $i \in \mathcal{N}$, the inter-execution times of all agents are lower bounded by a strictly positive lower bound.

Proof: The proof follows similar steps as the one of Theorem 1. In particular, As long as

$$
z_{i}^{2}>\frac{\frac{\varepsilon_{i}\left|N_{i}\right|}{a}+\frac{\bar{w}}{2 \zeta}}{\left(1-\sigma_{i}\right)\left(1-a\left|N_{i}\right|-\zeta / 2\right)}
$$

for all $i \in \mathcal{N}$, we know that $\dot{V}<0$. Thus $V(t)<V(0)$ for all $t>0$ and this implies $\|L x\| \leq\|B\| \sqrt{V(0)}$, i.e., $\|L x\|$ remains bounded. Assume now that agent $i$ updates its control law at time $t^{*}$. Then $e_{i}\left(t^{*}\right)=0$ and $f_{i}\left(t^{*}\right) \leq-\varepsilon_{i}<$ 0 , so that $i$ cannot trigger again instantaneously. Now since in between $t^{*}$ and the next update time of $i$ we have that $\dot{e}_{i}=$ $-\dot{x}_{i}=-u_{i}-w_{i}$, we have $\left|e_{i}(t)\right| \leq \int_{t^{*}}^{t}\left(\left|u_{i}(s)\right|+\left|w_{i}(s)\right|\right) d s$ for all $t>t^{*}$ until the next update time of $i$.

From (14) we have that $\|e\|^{2}=\sum_{i}\left|e_{i}\right|^{2} \leq \sum_{i} \theta_{i} z_{i}^{2}+$ $\sum_{i} \varepsilon_{i}$. Using the notation $\bar{\theta}=\max _{i} \theta_{i}$ and $\bar{\varepsilon}=\sum_{i} \varepsilon_{i}$ the last equation yields $\|e\|^{2} \leq \bar{\theta}\|L x\|^{2}+\bar{\varepsilon}$. We can then derive $\left|u_{i}\right| \leq\|u\|=\|L(x+e)\| \leq\|L x\|+\|L\|(\sqrt{\bar{\theta}}|| L x \|+\sqrt{\bar{\varepsilon}})$, and, using $\|L x\| \leq\|B\| \sqrt{V(0)}$, we finally have

$$
\left.\left|u_{i}(t)\right| \leq\|B\| \sqrt{V(0)}(1+\|L\| \sqrt{\bar{\theta}})+\|L\| \sqrt{\bar{\varepsilon}}\right) \triangleq \bar{u}^{\prime}
$$

Since $\left|e_{i}(t)\right| \leq \int_{t^{*}}^{t}\left(\left|u_{i}(s)\right|+\left|w_{i}(s)\right|\right) d s$ for all $t>t^{*}$, we have $\left|e_{i}(t)\right| \leq\left(\bar{u}^{\prime}+\bar{w}\right)\left(t-t^{*}\right)$, for all $t>t^{*}$ before the next event for agent $i$ is triggered. The next event is not triggered before (14) is violated, and this cannot happen before $\left|e_{i}(t)\right|=\sqrt{\varepsilon_{i}}$. A lower bound for the next event time is thus given by $t-t^{*}=\frac{\sqrt{\varepsilon_{i}}}{\bar{u}^{\prime}+\bar{w}}$. Therefore the minimum lower bound for the next event time for all agents $i$ and all times $t$ is given by $t-t^{*}=\frac{\min _{i} \sqrt{\varepsilon_{i}}}{\bar{u}^{\prime}+\bar{w}}$, or using the standard notation for the inter-event times, we have

$$
t_{k+1}^{i}-t_{k}^{i}=\frac{\min _{i} \sqrt{\varepsilon_{i}}}{\bar{u}^{\prime}+\bar{w}}
$$

for all $i \in \mathcal{N}, k=0,1, \ldots \diamond$

The above analysis establishes the finite $\mathcal{L}_{2}$ gain stability of the system and the existence of a strictly bounded interexecution times for all the agents. A similar analysis with the one used in the end of the previous section can yield similar results on the convergence of the perturbed system.

\section{EXAMPLES}

The results of the previous Sections are depicted through computer simulations.
Consider a network of four agents whose Laplacian matrix is given by

$$
L=\left[\begin{array}{cccc}
1 & -1 & 0 & 0 \\
-1 & 3 & -1 & -1 \\
0 & -1 & 2 & -1 \\
0 & -1 & -1 & 2
\end{array}\right]
$$

Agents are driven by (1) in the first simulation, while they are driven by (12) in the second simulation. The two figures depict the error norm evolution of the same agent in each case. The first figure depicts the evolution of the error norm and the corresponding element of the vector $z=L x$ for the specific agent 4 in the case of the system (1), driven by (5),(9). One can observe that the inter-execution times are lower bounded. The same example is recapped with

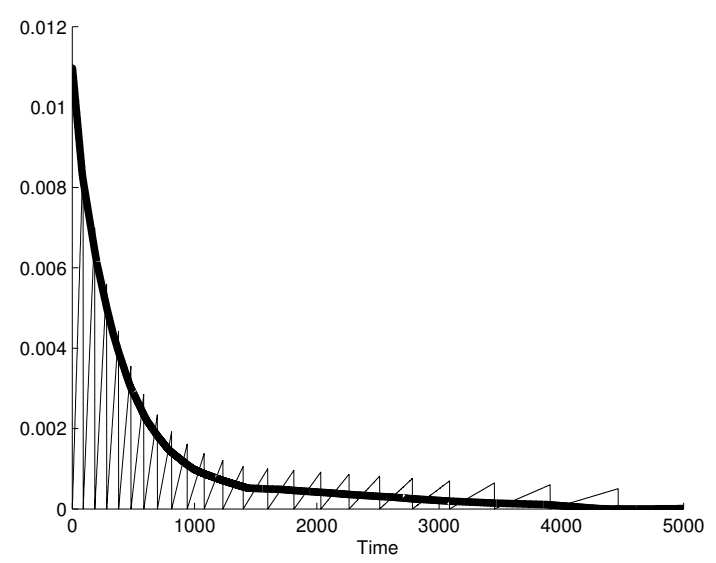

Fig. 1. Evolution of $\left|z_{4}\right|$ (bold line) and $\left|e_{4}\right|$ for the case of agents of the system (1), driven by (5),(9).

the addition of noise, as per (12), with the control design (5), (14). One can see that the event updates are still lower bounded, however the state trajectory is more distant than the desired equilibrium point $\left|z_{4}\right|=0$, due to the noise term. In both cases however, the trajectory remains within a bounded set from the agreement point, as expected from the derived results.

\section{CONClusions}

We extended our previous results on event-triggered agreement by proposing a triggering mechanism that is finite $\mathcal{L}_{2}$ gain stable with respect to additive disturbances in the open loop dynamics of the agents. Moreover, the control design is both distributed and provides strictly positive inter-execution times. Simulation examples support the derived theoretical results.

\section{REFERENCES}

[1] A.Anta and P.Tabuada. To sample or not to sample: self-triggered control for nonlinear systems. IEEE Transactions on Automatic Control, 55:2030-2042, 2010.

[2] M. Arcak. Passivity as a design tool for group coordination. IEEE Transactions on Automatic Control, 52(8):1380-1390, 2007.

[3] A. Arsie and E. Frazzoli. Efficient routing of multiple vehicles with no communications. International Journal of Robust and Nonlinear Control, 18(2):154-164, 2007. 


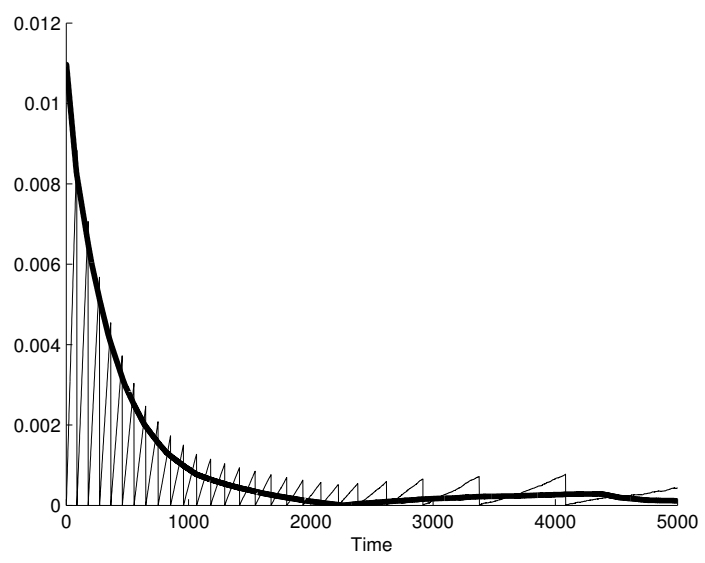

Fig. 2. Evolution of $\left|z_{4}\right|$ (bold line) and $\left|e_{4}\right|$ for the case of agents of the system (12), driven by (5)and update ruling (14) .

[4] M. Cao, B.D.O. Anderson, A.S. Morse, and C. Yu. Control of acyclic formations of mobile autonomous agents. 47th IEEE Conference on Decision and Control, pages 1187-1192, 2008.

[5] D.V. Dimarogonas and K.H. Johansson. Event-triggered control for multi-agent systems. 48th IEEE Conf. Decision and Control, pages 7131-7136, 2009.

[6] D.V. Dimarogonas and K.H. Johansson. Event-triggered cooperative control. European Control Conference, 2009.

[7] J.A. Fax and R.M. Murray. Graph Laplacians and stabilization of vehicle formations. 15th IFAC World Congress, 2002.

[8] C. Godsil and G. Royle. Algebraic Graph Theory. Springer Graduate Texts in Mathematics \# 207, 2001.

[9] W.P.M.H. Heemels, J.H. Sandee, and P.P.J. Van Den Bosch. Analysis of event-driven controllers for linear systems. International Journal of Control, 81(4):571-590, 2007.

[10] M. Ji and M. Egerstedt. Distributed coordination control of multiagent systems while preserving connectedness. IEEE Transactions on Robotics, 23(4):693-703, 2007.

[11] H. Khalil. Nonlinear Systems. Prentice-Hall, 2002.

[12] D. Lehmann and J. Lunze. Event-based control: A state feedback approach. European Control Conference, pages 1716-1721, 2009.

[13] R. Olfati-Saber and R.M. Murray. Consensus problems in networks of agents with switching topology and time-delays. IEEE Transactions on Automatic Control, 49(9):1520-1533, 2004.

[14] W. Ren and E.M. Atkins. Distributed multi-vehicle coordinated control via local information exchange. International Journal of Robust and Nonlinear Control, 17(10-11):1002-1033, 2007.

[15] G.S. Seyboth, D.V. Dimarogonas, and Karl H. Johansson. Control of multi-agent systems via event-based communication. 18th IFAC World Congress, 2011. to appear.

[16] A. Speranzon, C. Fischione, and K.H. Johansson. Distributed and collaborative estimation over wireless sensor networks. 45th IEEE Conference on Decision and Control, pages 1025-1030, 2006.

[17] P. Tabuada. Event-triggered real-time scheduling of stabilizing control tasks. IEEE Transactions on Automatic Control, 52(9):1680-1685, 2007.

[18] X. Wang and M. Lemmon. Finite-gain 12 stability in distributed eventtriggered networked control systems with data dropouts. European Control Conference, 2009.

[19] X. Wang and M.D. Lemmon. Event-triggered broadcasting across distributed networked control systems. American Control Conference, 2008.

[20] X. Wang and M.D. Lemmon. Self-triggered feedback control systems with finite-gain L2 stability. IEEE Transactions on Automatic Control, 45(3):452-467, 2009. 